\title{
On the Most General Linear Theory of Gravitation
}

\author{
Otto Nachtmann, Harald Schmidle, and Roman U. SexL \\ Institut für theoretische Physik, University of Vienna
}

Received December 12, 1967; in revised form March 5, 1969

\begin{abstract}
The most general field theory of gravitation is analyzed both group theoretically as well as physically. The field equations are solved by means of an algebraic method and it is found that any field theory of gravitation contains only one essential parameter which is correlated to the spin 0 content of the field. Further it turns out that any theory of gravitation must contain a nonvanishing spin 0 part, but general relativity is distinguished by the fact that its spin 0 component cannot be radiated.
\end{abstract}

After discovery of Einstein's general relativity numerous theories of gravitation have been proposed as alternatives to general relativity (see e.g. [1-4]). It can be shown [5] that all these theories are closely related to one another and differ from general relativity by the presence of a scalar component in the radiation field.

The most general quadratic Lagrangian for gravitation which can be built in terms of the field functions $\psi_{i k}$ and their first derivatives assuming $m_{\text {graviton }}=\emptyset$ is easily decomposed into its several spin parts. Using well known techniques from the theory of representations of the Poincaré group $[6,7]$ the Lagrangian

$$
\begin{gathered}
L(x)=L_{0}(x)+L^{\prime}(x), \\
L_{0}=\sum_{\alpha=1}^{4} c_{\alpha} I_{\alpha}, \quad L^{\prime}=f \psi_{i k} T^{i k} \\
I_{1}=\psi_{i k, l} \psi^{i k, l} ; \quad I_{2}=\psi_{i k, l} \psi^{i l, k} ; \quad I_{3}=\psi,{ }_{, k} \psi,^{k} ; \quad I_{4}=\psi, k \psi_{, l} l
\end{gathered}
$$

( $f$ coupling constant, $T_{i \text { i }}$ energy-momentum tensor of external mass fields, sum convention is adopted, $\psi=\psi_{k}^{k}$ ) can be decomposed into irreducible parts noticing that a symmetric tensor field $\psi_{i k}=\psi_{k i}$ transforms as

$$
\left[D\left(\frac{1}{2}, \frac{1}{2}\right) \times D\left(\frac{1}{2}, \frac{1}{2}\right)\right]_{\text {symmetric part }}=D(1,1)+D(0,0),
$$

where $D(1 / 2,1 / 2)$ is the vector representation of the Lorentz group. From there one sees that $\psi_{i k}$ contains four spin parts, namely one of spin 2 and spin 1, resp., as well as two spin 0 parts ${ }^{1}$. The Lagrangian

1 For details see [8] where we also give a technique to calculate projection operators for the irreducible spin parts for fields having arbitrary discrete spin. 
$L(x)$ may easily be transformed into momentum space by means of the action integral

$$
W=\int d^{4} x L(x)=\int d^{4} p \mathscr{L}(p) .
$$

$\mathscr{L}(p)$ is then given by

$$
\mathscr{L}(p)=p^{2} \psi^{*}\left[a_{2} P^{2}+a_{1} P^{1}+\sum_{\alpha, \beta=1}^{2} a_{\alpha \beta} P^{\alpha \beta}\right] \psi+f \psi_{i k}^{*} T^{i k} .
$$

$P^{1}$ and $P^{2}$ are projection operators into the spin 1 and the spin 2 subspace, respectively. $P^{\alpha \beta}$ are operators singling out spin 0 parts. They satisfy

$$
P^{\alpha \gamma} P^{\gamma^{\prime} \beta}=\delta \gamma \gamma^{\prime} P^{\alpha \beta}, 2 \quad P^{\alpha \beta}=x_{\alpha}^{0} x_{\beta}^{0},
$$

and the $x_{\alpha}^{0}$ transform as scalars.

The a's in (6) are constants which are fixed once the constants $c$ in Eq. (2) are given.

Varying the Lagrangian (6) one obtains after a suitable projection the four spin "separated field equations

$$
\begin{aligned}
2 a_{2} p^{2} P^{2} \psi & =-f P^{2} T=-f\left[T_{i k}-\frac{1}{3}\left(\eta_{i k}-\frac{p_{i} p_{k}}{p^{2}}\right) T\right], \\
2 a_{1} p^{2} P^{1} \psi & =0, \\
2 p^{2}\left(a_{11} x_{1}^{0}+a_{12} x_{2}^{0}\right) \psi & =0, \\
2 p^{2}\left(a_{21} x_{1}^{0}+a_{22} x_{2}^{0}\right) \psi & =-f x_{2}^{0} T=-\frac{1}{\sqrt{3}} f T .
\end{aligned}
$$

Eqs. (10) and (11) may ay be diagonalized to give

$$
p^{2}\left(P_{1}^{0}+P_{2}^{0}\right) \psi=-\frac{f}{6} \frac{1}{\operatorname{det} a_{\alpha \beta}} \frac{1}{p^{2}}\left[\eta_{i k} a_{11}-\frac{p_{i} p_{k}}{p^{2}}\left(a_{11}+\sqrt{3} a_{12}\right)\right] T .
$$

Then by using the completeness relation

$$
P^{2}+P^{1}+P_{1}^{0}+P_{2}^{0}=E,
$$

$E$ being the unit operator, one gets from Eqs. (8), (9), and (12) the following solution

$$
\psi_{i k}(p)=\psi_{i k}^{i n}(p) \delta\left(p^{2}\right)-f \frac{1}{p^{2}-i \varepsilon}\left[T_{i k}-k_{1} \eta_{i k} T+k_{2} \frac{p_{i} p_{b}}{p^{2}} T\right]
$$

which corresponds to a retarded Green's function nonvanishing only at the light cone. The constant $k_{1}$ is given by

$$
k_{1}=\frac{1}{3}\left(1-a_{2} \frac{a_{11}}{\operatorname{det} a_{\alpha \beta}}\right) \text {. }
$$

It can be shown that the constant $k_{2}$ does not possess any physical significance at all. (For a full discussion of the possible physical relevance of $k_{2} \mathrm{cf}$. [8].) Therefore it turns out that any field theory of gravitation

\footnotetext{
2 Explicite expressions for the P's are given in [8].
} 
is completely determined by two constants: The coupling constant and a further constant which is intimately connected with the spin 0 part contained in the field.

A closing remark regards general relativity. The Lagrangian which is obtained assuming invariance under gauge transformations

$$
\psi_{i k} \rightarrow \psi_{i k}+p_{k} \Lambda_{i}+p_{i} \Lambda_{k}
$$

is uniquely determined if one makes the trivial requirement that the Lagrangian can be locally formulated in $x$-space. This Lagrangian is (up to first order) identical with that of general relativity. The interesting fact which turns out is that general relativity is not a pure spin 2 theory. It contains a spin 0 component which, however, cannot be radiated.

\section{References}

1. Jordan, P.: Z. Physik 157, 112 (1960).

2. Brans, C., and R. Dicke: Phys. Rev. 124, 925 (1961).

3. Capella, A.: N. Cim. 42 B, 321 (1960).

4. Belinfante, F., and J. Swihart: Ann. Phys. (N.Y.) 1, 168 (1957).

5. SExu, R.: Fortschr. Phys. 15, 269 (1965).

6. Wigner, E.: Ann. Math. 40, 149 (1939).

7. Joos, H.: Fortschr. Phys. 10, 65 (1962).

8. Nachtmann, O., H. Schmidle, and R. Sexu: Acta Phys. Austr. 29, Heft 4 (1969).

O. NachtmanN Theory Division CERN

CH-1211 Genf 23
H. SCHMTDLE

III. Physikalisches Institut der Technischen Hochschule 5100 Aachen, Charlottenstraße 14

R. U. SEXL

Institut für theoret. Physik der Universität

A 1090 Wien

Boltzmanngasse 5 